\title{
Tissue-Specific mRNA Expression Profiles of Human Carbohydrate Sulfotransferase and Tyrosylprotein Sulfotransferase
}

\author{
Masuhiro NishimURA* and Shinsaku NAITO \\ Division of Pharmacology, Drug Safety and Metabolism, Otsuka Pharmaceutical Factory, Inc.; 115 Tateiwa, Muya-cho, \\ Naruto, Tokushima 772-8601, Japan.
}

Received October 11, 2006; accepted January 19, 2007; published online January 22, 2007

Pairs of forward and reverse primers and TaqMan probes specific to each of 15 human sulfotransferases were prepared. The mRNA expression level of each target enzyme was analyzed in total RNA from single and pooled specimens of various human tissues (adrenal gland, bone marrow, brain, colon, heart, kidney, liver, lung, pancreas, peripheral leukocytes, placenta, prostate, salivary gland, skeletal muscle, small intestine, spinal cord, spleen, stomach, testis, thymus, thyroid gland, trachea, and uterus) by real-time reverse transcription PCR using an ABI PRISM 7700 Sequence Detection System. The mRNA expression profiles of the sulfotransferases in these 23 different human tissues were used to identify the tissues exhibiting high transcriptional activity for these enzymes. These results provide valuable information for studies concerning the human carbohydrate sulfotransferase and tyrosylprotein sulfotransferase genes in various tissues.

Key words sulfotransferase; mRNA expression; tissue distribution; quantification

L-Selectin ligands require sulfation as a post-translational modification. ${ }^{1)}$ Sulfotransferases potentially involved in the generation of selectin ligands have recently been reported. $^{2-5)}$ These enzymes, such as carbohydrate sulfotransferase (CHST) and tyrosylprotein sulfotransferase (TPST), can sulfate the carbohydrate and protein moieties, respectively. Li et al. have reported that CHST1 and CHST2 contribute to the generation of optimal L-selectin ligands in vascular endothelial cells at sites of inflammation. ${ }^{6)}$ Hiraoka et al. have reported that CHST8 and CHST9 play critical roles in forming sulfo $\rightarrow 4$ GalNAc $\beta 1 \rightarrow 4 \mathrm{GlcNAc} \beta 1 \rightarrow \mathrm{R}$ in both $N$ glycans and $O$-glycans in a tissue-specific manner. ${ }^{7)}$ Hiraoka et al. have also reported that CHST11 and CHST12 play complementary roles in chondroitin and dermatan sulfate synthesis in different tissues. ${ }^{8)}$ Ouyang and Moore have reported that TPST-1 and TPST-2 show distinct specificities toward peptide substrates. ${ }^{5)}$ Thus, the differences in substrate specificity of sulfotransferases have been demonstrated in a number of studies. However, there is only very limited information concerning the relationships between the expres- sion level and function of human sulfotransferases because the molecular cloning of human sulfotransferases as reported in the present study was not achieved until 1997 to $2001^{2-5,7-16)}$ and studies on these sulfotransferases are therefore still in the early stages. The tissue distributions of many sulfotransferases such as CHST1, ${ }^{9}$ CHST3, ${ }^{3)}$ CHST4, ${ }^{2)}$ CHST5, ${ }^{11)}$ CHST $7,{ }^{13)}$ CHST9, ${ }^{7)}$ TPST $1,{ }^{4)}$ and TPST2 ${ }^{5)}$ have also been reported. However, the tissue distribution of the mRNA expression of large numbers of human sulfotransferase genes has not been evaluated under the same experimental conditions using relatively quantitative data. The present study was therefore undertaken to investigate the mRNA expression levels of 15 human sulfotransferases using high-sensitivity real-time reverse transcription PCR (RTPCR) in total RNA from single and pooled specimens of 23 adult human tissues. The nomenclature of these sulfotransferases is summarized in Table 1.

Table 1. Nomenclature of Sulfotransferases

\begin{tabular}{llll}
\hline \hline \multicolumn{1}{c}{ Name } & Abbreviation & $\begin{array}{c}\text { GenBank } \\
\text { accession No. }\end{array}$ & \multicolumn{1}{c}{ Trivial name(s)/synonym(s) } \\
\hline Carbohydrate (keratan sulfate Gal-6) sulfotransferase 1 & CHST1 & NM_003654 & C6ST, KS6ST, KSGa16ST \\
Carbohydrate $(N$-acetylglucosamine-6- $O$ ) sulfotransferase 2 & CHST2 & NM_004267 & C6ST \\
Carbohydrate (chondroitin 6) sulfotransferase 3 & CHST3 & NM_004273 & C6ST, C6ST1 \\
Carbohydrate $(N$-acetylglucosamine 6- $O$ ) sulfotransferase 4 & CHST4 & NM_005769 & LSST, HEC-GlcNAc6ST \\
Carbohydrate $(N$-acetylglucosamine 6- $O$ ) sulfotransferase 5 & CHST5 & NM_012126 & ALYE870, PRO1886, FLJ22167, MGC74625 \\
Carbohydrate $(N$-acetylglucosamine 6- $O$ ) sulfotransferase 6 & CHST6 & NM_021615 & MCDC1 \\
Carbohydrate $(N$-acetylglucosamine 6- $O$ ) sulfotransferase 7 & CHST7 & NM_019886 & C6ST-2 \\
Carbohydrate $(N$-acetylgalactosamine 4-0) sulfotransferase 8 & CHST8 & NM_022467 & GALNAC4ST-1 \\
Carbohydrate $(N$-acetylgalactosamine 4-0) sulfotransferase 9 & CHST9 & NM_031422 & GALNAC4ST-2 \\
Carbohydrate sulfotransferase 10 & CHST10 & NM_004854 & HNK1ST, HNK-1ST, MGC17148 \\
Carbohydrate (chondroitin 4) sulfotransferase 11 & CHST11 & NM_018413 & C4ST, C4ST1, C4ST-1, HSA269537 \\
Carbohydrate (chondroitin 4) sulfotransferase 12 & CHST12 & NM_018641 & C4S-2, C4ST2, C4ST-2 \\
Galactose-3- $O$-sulfotransferase 1 & GAL3ST1 & NM_004861 & CST \\
Tyrosylprotein sulfotransferase 1 & TPST1 & NM_003596 & \\
Tyrosylprotein sulfotransferase 2 & TPST2 & NM_003595 & \\
\hline
\end{tabular}


Table 2. Total RNA Source Information for Various Tissues

\begin{tabular}{|c|c|c|c|c|}
\hline Tissue & Pool size & Age & Sex & Race \\
\hline Adrenal gland & 61 & $15-61$ years & Female, male & Caucasian \\
\hline Bone marrow & 8 & $18-56$ years & Female, male & Caucasian \\
\hline Brain & 1 & 43 years & Male & Caucasian \\
\hline Colon & 1 & 35 years & Female & Caucasian \\
\hline Heart & 10 & $21-51$ years & Female, male & Caucasian \\
\hline Kidney & 14 & $18-59$ years & Female, male & Caucasian \\
\hline Liver & 1 & 51 years & Male & Caucasian \\
\hline Lung & 3 & $15-40$ years & Female, male & Caucasian \\
\hline Pancreas & 2 & 50,55 years & Male & Caucasian \\
\hline Peripheral leukocytes & 250 & $18-40$ years & Female, male & Caucasian \\
\hline Placenta & 8 & $19-39$ years & Female & Caucasian \\
\hline Prostate & 32 & $21-50$ years & Male & Caucasian \\
\hline Salivary gland & 24 & $15-60$ years & Female, male & Caucasian \\
\hline Skeletal muscle & 7 & $20-68$ years & Female, male & Caucasian \\
\hline Small intestine & 5 & $20-61$ years & Female, male & Caucasian \\
\hline Spinal cord & 49 & $15-66$ years & Female, male & Caucasian \\
\hline Spleen & 14 & $30-66$ years & Female, male & Caucasian \\
\hline Stomach & 1 & 50 years & Male & Caucasian \\
\hline Testis & 39 & $14-64$ years & Male & Caucasian \\
\hline Thymus & 3 & $20-38$ years & Male & Caucasian \\
\hline Thyroid gland & 65 & $15-61$ years & Female, male & Caucasian \\
\hline Trachea & - & $18-54$ years & Female, male & Caucasian \\
\hline Uterus & 3 & $40-61$ years & Female & Caucasian \\
\hline
\end{tabular}

-, no data available.

\section{MATERIALS AND METHODS}

Materials Total RNA prepared from single and pooled specimens of adult human tissues (adrenal gland, bone marrow, brain, colon, heart, kidney, liver, lung, pancreas, peripheral leukocytes, placenta, prostate, salivary gland, skeletal muscle, small intestine, spinal cord, spleen, stomach, testis, thymus, thyroid gland, trachea, and uterus) was purchased from CLONTECH Laboratories, Inc. (Hilden, Palo Alto, CA, U.S.A.). The total RNA source information for these human tissues is shown in Table 2. The total RNA of the 23 adult human tissues (Table 2) used in the present study was the same as that used for the measurement of the mRNA expression levels of transporters in our previous studies. ${ }^{17,18)}$ The present study was approved by the Ethics Committee of Otsuka Pharmaceutical Factory, Inc. Yeast tRNA was purchased from Life Technologies, Inc. (Rockville, MD, U.S.A.), and the TaqMan One-Step RT-PCR Master Mix Reagents Kit was purchased from Applied Biosystems (Foster City, CA, U.S.A.). All other chemicals used in this study were of reagent grade.

Oligonucleotides The pair of forward and reverse primers and the TaqMan probe for peptidylprolyl isomerase A (PPIA) used for RT-PCR analysis employed sequences that have been reported previously. ${ }^{19}$ The pairs of forward and reverse primers and the TaqMan probes for CHST1, CHST3, CHST4, CST, and TPST2 used for RT-PCR analysis also employed sequences that have been reported previously. ${ }^{20)}$ The pair of forward and reverse primers and the TaqMan probe for TPST1 used for RT-PCR analysis also employed sequences that have been reported previously. ${ }^{21)}$ The pairs of primers and the TaqMan probes for the target mRNAs were designed based on the human mRNA sequence (Table 3) using Primer Express software (Applied Biosystems). The GenBank accession number and the position from the initia-

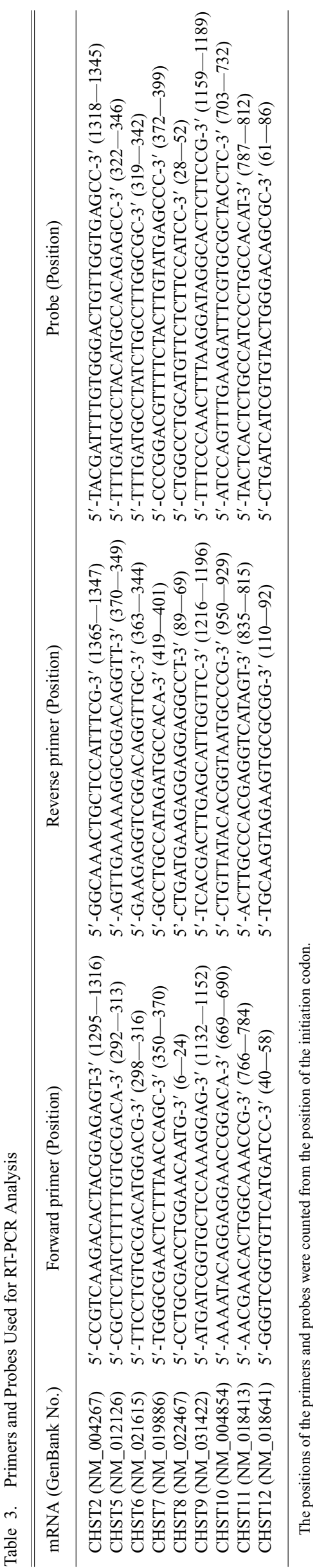


Table 4. Expression of Human Sulfotransferase mRNAs in Various Tissues

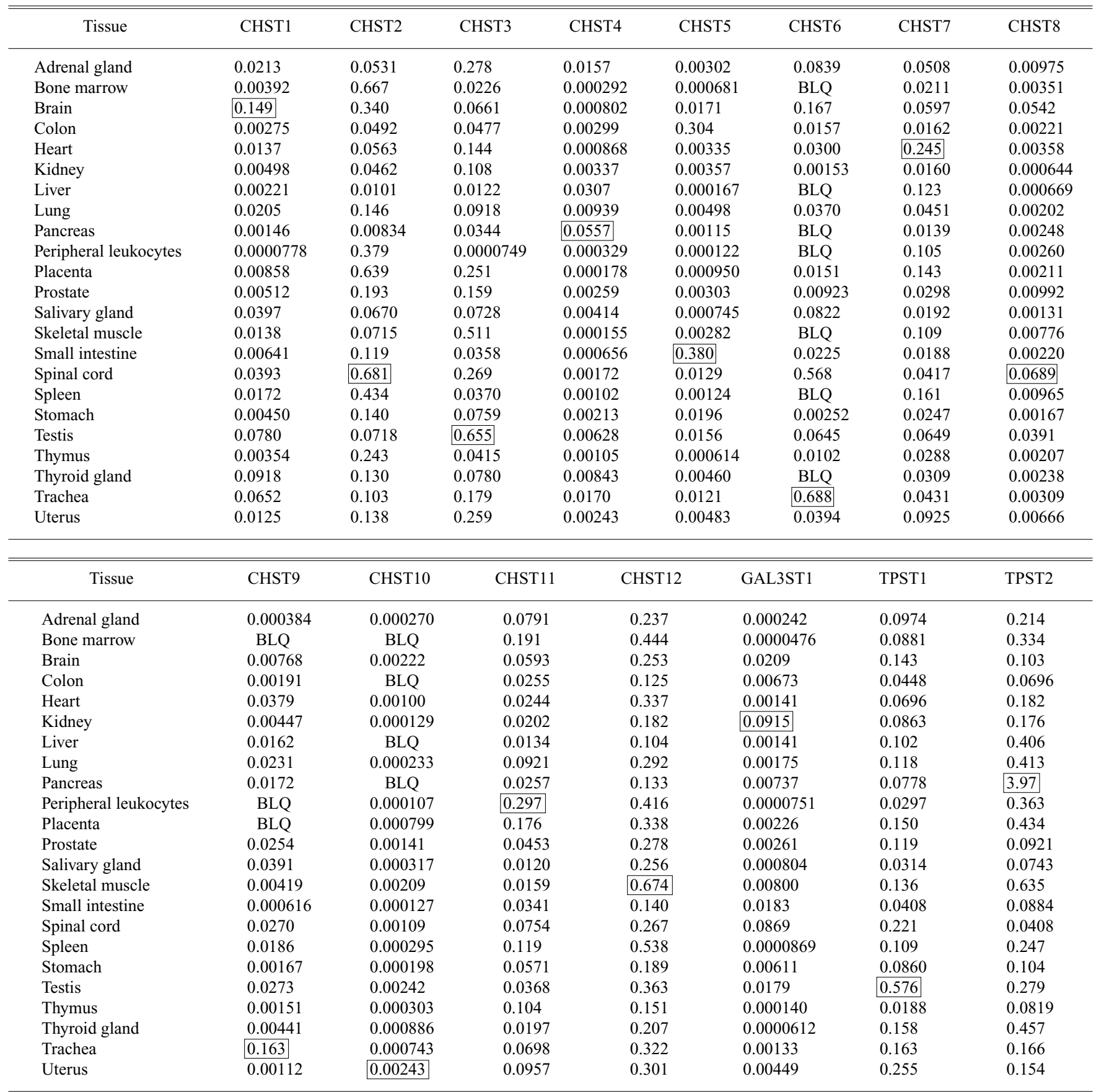

BLQ, below the limit of quantification. Data are expressed as the ratio of the target mRNA to PPIA mRNA. Experiments were performed in duplicate. The highest values among the various tissues are shown in boxes.

tion codon are also shown in Table 3. Each primer and/or probe was homology searched by an NCBI BLAST search to ensure that it was specific for the target mRNA transcript. The primers and TaqMan probes were synthesized by QIAGEN Japan (Tokyo, Japan) and the Genosys Division of SIGMA-ALDRICH JAPAN K.K. (Ishikari, Hokkaido, Japan). The TaqMan probes contained 6-carboxyfluorescein (FAM) at the $5^{\prime}$ end and 6-carboxytetramethylrhodamine (TAMRA) at the $3^{\prime}$ end and were designed to hybridize to a sequence located between the PCR primers.
TaqMan RT-PCR Conditions Total RNA samples obtained from adult human tissues were diluted under the same conditions as in our previous studies. ${ }^{17,18)}$ Total RNA at $25000 \mathrm{pg}$ per $50 \mu \mathrm{l}$ of reaction mixture was used for measurement of the target mRNA in each tissue. The RT-PCR assay was performed using the ABI PRISM 7700 Sequence Detection System (Applied Biosystems) under the same conditions as in our previous studies. ${ }^{17,18)}$

Data Analysis Samples were deemed positive at any given cycle when the value of the emitted fluorescence was 
greater than the threshold value calculated by the instrument's software (Sequence Detector Ver. 1.9.1). The threshold cycle $(\mathrm{Ct})$, which is defined as the cycle at which PCR amplification reaches a significant value (i.e., usually 15 times greater than the standard deviation of the baseline), is given as the mean value. The relative expression of each mRNA was calculated by the $\Delta \mathrm{Ct}$ method (where $\Delta \mathrm{Ct}$ is the value obtained by subtracting the $\mathrm{Ct}$ value of PPIA mRNA from the $\mathrm{Ct}$ value of the target mRNA), as employed in our previous studies. ${ }^{17,18)}$ Specifically, the amount of target mRNA relative to PPIA mRNA is expressed as $2^{-(\Delta \mathrm{Ct})}$. Data are expressed as the ratio of the target mRNA to PPIA mRNA.

\section{RESULTS AND DISCUSSION}

In this study, we investigated the mRNA expression levels of 15 human sulfotransferases in adult human tissues. Analysis was conducted by RT-PCR using the ABI PRISM 7700 Sequence Detection System in the presence of the TaqMan probe. We selected PPIA as the endogenous control for mRNA measurements. The rationale used to select the most suitable housekeeping gene has been described in detail in our previous studies. ${ }^{17,19,22)}$ The mRNA expression levels of human CHSTs, GAL3ST1, and TPSTs in various tissues are shown in Table 4. Consistent with the findings of another study in human subjects, ${ }^{9)}$ CHST1 mRNA was expressed ubiquitously, with the highest levels of expression in the brain and very low levels of expression in peripheral leukocytes. CHST2 mRNA was expressed ubiquitously, with the highest levels of expression in the spinal cord. Similar to the findings of another study in human subjects, ${ }^{3)}$ CHST3 mRNA was expressed ubiquitously, with the highest levels of expression in the testis and very low levels of expression in peripheral leukocytes. Similar to the findings of another study in human subjects, ${ }^{2)}$ CHST4 mRNA was expressed ubiquitously, with high levels of expression in the liver and pancreas as compared with other tissues. Consistent with the findings of another study in human subjects, ${ }^{11}$ CHST5 mRNA was expressed ubiquitously, with high levels of expression in the colon and small intestine. CHST6 mRNA was expressed at high levels in the brain, spinal cord, and trachea and was detectable in the adrenal gland, colon, heart, kidney, lung, placenta, prostate, salivary gland, small intestine, stomach, testis, thymus, and uterus. Similar to the findings of another study in human subjects, ${ }^{13)}$ CHST7 mRNA was expressed ubiquitously, with the highest levels of expression in the heart. Consistent with the findings of other studies in human subjects, ${ }^{7,14}$ CHST8 mRNA was expressed ubiquitously, with high levels of expression in the brain, spinal cord, and testis. Consistent with the findings of another study in human subjects, ${ }^{7)}$ CHST9 mRNA was expressed at the highest levels in the trachea and was detectable in other tissues except for the bone marrow, peripheral leukocytes, and placenta. Similar to the findings of another study in human subjects, ${ }^{15)}$ CHST10 mRNA was expressed at high levels in the brain, skeletal muscle, testis, and uterus and was detectable in other tissues except for the bone marrow, colon, liver, and pancreas. Consistent with the findings of another study in human subjects, ${ }^{8)}$ CHST11 and CHST12 mRNA were expressed ubiquitously, with the highest levels of ex- pression in peripheral leukocytes and skeletal muscle, respectively. GAL3ST1 mRNA was expressed ubiquitously, with the highest levels of expression in the kidney and very low levels of expression in the bone marrow, peripheral leukocytes, spleen, and thyroid gland. Consistent with the findings of another study in human subjects and mice, ${ }^{4)}$ TPST1 mRNA was expressed ubiquitously, with the highest levels of expression in the testis. Consistent with the findings of another study in human subjects and mice, ${ }^{5)}$ TPST2 mRNA was expressed ubiquitously, with the highest levels of expression in the pancreas. The results for the tissue-specific mRNA expression profiles of each human CHST and TPST are summarized by comparison of qualitative data such as that obtained by Northern blotting analysis against data reported in the literature. However, there is only very limited information concerning the relationships between the expression site and function because studies on these sulfotransferases are still in the early stages. We therefore hope that other researchers will find our results helpful in conducting studies to clarify the relationships between the expression site and function.

In conclusion, the results of the present study provide valuable information concerning the tissue-specific profiles of mRNA expression of human carbohydrate sulfotransferase and tyrosylprotein sulfotransferase in various tissues. The information obtained in this study may be important in assessing the feasibility of measuring the mRNA expression levels of target genes in target tissues.

Acknowledgments The authors would like to thank David G. Spear, M.D., for reviewing this manuscript.

\section{REFERENCES}

1) Hemmerich S., Butcher E. C., Rosen S. D., J. Exp. Med., 180, 2219 2226 (1994).

2) Bistrup A., Bhakta S., Lee J. K., Belov Y. Y., Gunn M. D., Zuo F. R., Huang C. C., Kannagi R., Rosen S. D., Hemmerich S., J. Cell Biol., 145, 899-910 (1999).

3) Fukuta M., Kobayashi Y., Uchimura K., Kimata K., Habuchi O., Biochim. Biophys. Acta, 1399, 57-61 (1998).

4) Ouyang Y. B., Lane W. S., Moore K. L., Proc. Natl. Acad. Sci. U.S.A., 95, 2896-2901 (1998).

5) Ouyang Y. B., Moore K. L., J. Biol. Chem., 273, 24770-24774 (1998).

6) Li X., Tu L., Murphy P. G., Kadono T., Steeber D. A., Tedder T. F., J. Leukoc. Biol., 69, 565-574 (2001).

7) Hiraoka N., Misra A., Belot F., Hindsgaul O., Fukuda M., Glycobiology, 11, 495-504 (2001).

8) Hiraoka N., Nakagawa H., Ong E., Akama T. O., Fukuda M. N., Fukuda M., J. Biol. Chem., 275, 20188-20196 (2000).

9) Fukuta M., Inazawa J., Torii T., Tsuzuki K., Shimada E., Habuchi O., J. Biol. Chem., 272, 32321-32328 (1997).

10) Li X., Tedder T. F., Genomics, 55, 345-347 (1999).

11) Lee J. K., Bhakta S., Rosen S. D., Hemmerich S., Biochem. Biophys. Res. Commun., 263, 543-549 (1999).

12) Uchimura K., Fasakhany F., Kadomatsu K., Matsukawa T., Yamakawa T., Kurosawa N., Muramatsu T., Biochem. Biophys. Res. Commun., 274, 291-296 (2000).

13) Kitagawa H., Fujita M., Ito N., Sugahara K., J. Biol. Chem., 275, $21075-21080$ (2000).

14) Xia G., Evers M. R., Kang H. G., Schachner M., Baenziger J. U., J. Biol. Chem., 275, 38402-38409 (2000).

15) Ong E., Yeh J. C., Ding Y., Hindsgaul O., Fukuda M., J. Biol. Chem., 273, 5190-5195 (1998).

16) Honke K., Tsuda M., Hirahara Y., Ishii A., Makita A., Wada Y., J. Biol. Chem., 272, $4864-4868$ (1997). 
17) Nishimura M., Naito S., Drug Metab. Pharmacokinet., 20, 452-477 (2005).

18) Nishimura M., Naito S., Drug Metab. Pharmacokinet., 21, 357-374 (2006).

19) Nishimura M., Naito S., Yokoi T., Drug Metab. Pharmacokinet., 19, 135-149 (2004).
20) Nishimura M., Yoshitsugu H., Naito S., Hiraoka I., Yakugaku Zasshi, 122, 339-361 (2002)

21) Nishimura M., Yoshitsugu H., Yokoi T., Tateno C., Kataoka M., Horie T., Yoshizato K., Naito S., Xenobiotica, 35, 877-890 (2005).

22) Nishimura M., Naito S., Biol. Pharm. Bull., 28, 886-892 (2005). 